\title{
Modelling Semiosis of Design
}

\author{
V.V. Kryssanov \\ Dept. Computer and Systems Engineering, Kobe University,Rokkodai, Nada-ku, Kobe 657- \\ 8501, Japan \\ E-m: kryssanov@al.cs.kobe-u.ac.jp \\ J.B.M. Goossenaerts \\ Faculty of Technology Management, Eindhoven University of Technology, The Netherlands \\ E-m:J.B.M.Goossenaerts@tm.tue.nl
}

Keywords Design, Semiosis, Information Infrastructure

\begin{abstract}
This paper addresses the modelling of the cognitive and evolutionary aspects of engineering design in support of two broad goals: the better understanding of product life cycle processes, and the development of relevant information infrastructure services. The modelling elements are derived from the Framework of Industrial Semiosis. The semiosis of evolutionary design is explained, and general principles of the evolution of the product concept are formulated.
\end{abstract}

\section{INTRODUCTION}

As products and production processes become more specific to individual requirements, there is a need to expand and diversify information infrastructure services for design and configuration of products. The development of these services requires a thorough understanding of the process in which the product evolves as driven by the customer's needs and desires, the environment of use, the designer's imagination, and the enterprise strategic goals and capabilities.

Our expectations for the information infrastructure services for design processes have, over a long time, been guided by evolutions in supply chains. These show a clear trend towards strongly differentiated logistics processes that are realized after reconstructing the sales and fulfillment cycle, whereby the traditional production process is broken down and reconstructed in a manner that maximizes the overall efficiency of the chain [18]. This trend will be enhanced by advanced electronic commerce systems, 
virtual organizations, and e-markets, enabling enterprises, small and large, to offer their capabilities in multifarious commercial models [1].

The large variety of products, customer desires, and use-environments calls for a similar differentiation in design semiosis processes [6]. In these processes, the product is determined (conceived-represented-realized) by devising a product structure and the possible relations between the product and its operating environment, which together aggregate the possible product configurations [13]. Most naturally, the product and its environment are defined in terms of a product language subject to the semiosis processes. The dependence of the semiosis of design both on the entire product life cycle and on the dynamic (economical, social, technical, etc.) environments is critical for organizing efficient computer-aided support for the development of modern high tech products.

The goal of the present work is to advance the modelling of design semiosis. The resulting models serve as an input for the creation of infrastructure services for evolutionary design. They also offer an improved understanding of the design process in a context of the product life cycle.

\section{PARADIGMS OF DESIGN}

A paradigm may be seen as a disciplinary matrix that $(a)$ has three main properties: common symbolic abstractions, model beliefs, and a system of values; and $(b)$ that generalizes some examples, which illustrate the properties. Taking this definition as a basis, we will briefly review three most influential design paradigms learnt from the literature.

In the Empirical Paradigm, a design theory is to be founded on empirical knowledge and factual data. Such a theory usually has a methodological character and focuses on systematizing, structuring, and ordering of both design problems and problem solving methods. The Empirical Paradigm is based on a quite naïve assumption that all the design problems are well structured, and that there exists ideal knowledge for every given problem. The works [19] and [10] are typical representatives of this approach: their strong point is that if a task can be classified and related to the accepted system of beliefs and values, the formulated principles can guide the designer throughout the problem solving process. Unfortunately, the class of design tasks that would be solved in this way, although important, is very limited [2].

The Logical Paradigm attempts to compensate for the limitations of the empirical approach. Its fundamental assumption is about the possibility of decompositing a complex design problem into a number of sub-problems with relatively independent goals. In this paradigm, the design process starts 
from analysis of requirements, passes through one or more stages of synthesis directed by the decomposition, and is completed by evaluation. If the obtained outcome is found unsatisfactory, the process may be repeated. [11] and [16] present design theories that propose some principles for systematization of the synthesis-analysis interaction while designing. The fallacy, however, is that design problems are often incomplete as the goals are only partially understood, and solutions are emergent rather than decomposed. Besides, the requirements may be inconsistent, and during the synthesizing of the decomposed solutions, second order requirements may arise due to the interaction of the solutions. Many design theories, which come from Artificial Intelligence (e.g. [2]), reveal similar drawbacks: in practice, not only the initial problem state, but also the goal state is frequently ill defined, and it is generally infeasible to a priory assign a system of operators (rules, synthesis-analysis procedures, etc.) for the transitions from the initial state to the goal state. Overall, the Logical Paradigm suggests a rather straightforward view of the design process and can only deal with design problems of a low complexity.

The Evolutionary Design Paradigm explicitly recognizes the social, evolutionary, and error-prone nature of product development. It postulates the tentative and conditional character of design solutions and strives first to develop the main scientific body of design philosophy. The life-cycle design approaches realize some of the important aspects of this Paradigm by making the adequacy of the design dependent on the requirements, which are not constant but change over time [17]. Evolutionary Design is more general and assimilates the earlier theories. However, because of the complexity and multi-disciplinary nature, Evolutionary Design is still not properly framed, while the different 'evolutionary' approaches remain isolated at the level of computer applications and cannot deal with the dynamic aspects of the design process [9]. In the following section, an approach is proposed that could address the principal weaknesses of the Evolutionary Paradigm.

\section{MODELLING EVOLUTIONARY DESIGN}

\subsection{Semiotics}

Thinking in engineering design requires and is based on the representation and processing of information in the mind, prior to any physical realization of the design [12]. Semiotics - a science about signs, signification, and sign systems - provides a powerful and elegant tool for the investigation of thought and evolutionary processes involving products and their operational environments. Semiotics deals with an '...action, or 
influence, which is, or involves, an operation of three subjects, such as a sign, its object and its interpretant, this tri-relative influence not being in any way resolvable into an action between pairs' [3].

Semiotics studies how signs mediate meaning through the processes of semiosis, in which something functions as a sign. Semiosis is classically defined on and affects the signifier (the sign vehicle or token; usually a sign), the signified (the designatum, e.g. an object, whether physical or abstract), and the interpretant (i.e. the sense made of the sign or that which follows semantically from the process of interpretation). The semiosis of design is modelled by sign systems standing in for conceptual spaces and physical phenomena. These sign systems are evolved, blended, and analyzed, resulting in the creation of new concepts and the design itself [12].

\subsection{The Product in its Environment}

In the semiotic approach, human cognition at any stage of the product life cycle is characterized as a structuring of experience and perception to provide structured information - a language - that is to deal with the product. A design is seen as a text 'written' in such a product language with a syntax, which constrains the product's topological organization, semantics, which defines the functional structure and product-environment interaction, and pragmatics, which manifests physiological, psychological, and sociological effects associated with the product. This language is a sign system that influences and, conditional on the usage context, determines the meaning conveyed with signs. The sense made of a sign is understood as also a sign in the interpreter's mind.

A product is normally perceived through its distinctions that are revealed as technologic, contextual, and ergonomic relations between product parts and between the product and its environment, which includes consumers. The relations are represented in a language. In every situation, the language (that is a system of signs) may be different but has a common ground - the reality - that fundamentally constrains the relations. The language does not prescribe a particular model, but instead provides us with a universe of models for physical and mental phenomena.

The Artifactual Wheel-Work (AWW) classifies product life-cycle related models and techniques [7]. The framework postulates that, as one observes different levels of progression and repetition in the human dealing with artifacts, a natural distinction should be made between 3 kinds of existence in the life cycle of products and their groupings as follows: the individuals or ens (e.g. a single product), giving rise to the ontogenic wheel; types (e.g. the type of the product) or generations (in the case of self-reproducing species), 
giving rise to the typogenic wheel; and tribes or families (phylos), e.g. the totality of previous and actual types and occurrences of the product, or of all products, giving rise to the phylogenic wheel.

The distinctions between ens, types, and phylos, explain the existence of different models: an ontogenic model presenting life history data and future data about an occurrence of an artifact type; a typogenic model combining architectural and developmental aspects of an artifact type; and a phylogenic model integrating typogenic models of different generations, along with the steps that have been taken to move from one generation to another (e.g. reuse, production problem solving, etc). All three models matter for both the product and the environment. Design semiosis processes can naturally be classified as contributing to typogenesis, ontogenesis, or phylogenesis for the artifact, and resting on the genesis of 'resources' in the environment.

Design expectations are defined as stable relation patterns existing in the product language and realized in the product (see [13] for details). Violation of design expectations is the 'driving force' for design semiosis. As such, violations of functionality-related expectations control typogenesis, while violations of environment-related expectations activate phylogenesis in the product life cycle. Complementary to these, the law of Weber-Fechner, which tells us that in order for a series of quantities to appear 'regular' in our perception, it should be based on a geometric scale, characterizes the dynamics of the ontogenesis processes, where relation patterns are originally detected to be further abandoned or realized in the product [15].

Complementary to the AWW, and generalizing the concepts of resource and enterprise, the Enterprise wheel-work (EWW) describes the space-timematter situated industrial system of resources and their structures in enterprises [6]. These resources, during their ontogenic life cycles, play rôles in tasks moving the ontogenic, typogenic, or phylogenic wheels of artifacts.

With the artifact life cycle in focus, the resource concept can be broadened to also cover objects interacting with the product in its useenvironment. Thus, the EWW serves as a basis also for the modelling of elements in the product's use-environment. This environment can be considered a stable but evolving combination of 'resources' with which the product will interact. The environment offers the context within which the 'customer' exploits the product, and which - through its own evolution forms a source of new opportunities and threats for the product's evolution.

\subsection{Semiosis processes}

Consumers' needs are first detected as stable patterns of conceptual relations. An initial product concept is nothing but a composition of related 
signs. The signs can be arbitrary, while the evolution of the concept throughout the product life cycle can be described in terms of transitions between different relation patterns by means of a distinction dynamics [14].

The first fundamental law of design semiosis states that in the product life cycle, the distinction dynamics always seeks to decrease the number of possible relations between the product and its environment [13]. A more precise characterization of design semiosis can be done, however, by introducing four types of elementary transformations (translations) of the product language, reflecting conservation, destruction, creation, and creation and destruction cognitive processes [8]. Semiosis processes of the first type result in preserving certain elements of the product language and are completely controllable, predictable, and reversible. Instances of such processes are selection, optimization, any kind logical or causal inference, methodologies, and deterministic algorithms. The second type of process is, in contrast to the first, non-distinction conserving: there is an information loss affecting the language elements. Such a process is always not reversible but still predictable, e.g. abstraction, habituation, adaptation, and the like. Next, semiosis processes behind creation can well be described as adoption from 'the outside' (acquisition) of new elements to the product language. These processes may only statistically be predictable, but they can, in principle, be reconstructed in the present-to-past direction (e.g. analogical or metaphorical reasoning, discovery, or any type of emergence). Finally, the last class of semiosis processes involves both the creation and destruction ('forgetting') of product language elements. Such processes are not systematic in any way (e.g. trial-and-error experiences), and they are not reconstructable. While all these different semiosis processes can formally be defined in terms of Algebraic Semiotics at the quite computational level [4] [12], we limit our discussion to qualitative characterization of the product life cycle processes.

The Framework of Industrial Semiosis [6] distinguishes four activity layers - observation, operations, improvement, and invention and innovation - and corresponding contexts for industrial semiosis. This framework permits us to classify the AWW semiosis processes. At the observation layer, life cycle ontogenesis has to be, in the main, a conservation process. Indeed, each particular artifact is associated with certain distinctions; distinction change usually leads to consideration of a different (even if slightly) artifact. Conservation is also important in typo- and phylogenesis, although to a less extent. At the operational layer, only ontogenesis has a plain and unequivocal characterization: this type of semiosis processes have to be non-distinction conserving. By denying the latter, almost any artifact would become unmanageable at the cognitive level (due to the natural 
limitations on the interpreter's side), as its handling would every time require possessing the artifact' perfect and complete knowledge. The improvement layer, though it allows for some destruction, preserves artifact distinctions throughout the semiosis processes. The onto-, typo-, and phylogenesis differ there by the degree of the permitted destruction (from less to higher, respectively). Obviously, as we come to the invention and innovation layer, we can unambiguously consider only the case of ontogenesis, where the creation semiosis processes play the major rôle.

\section{CONCLUSION AND FUTURE WORK}

The semiotic theory of evolutionary design has been developed into a more detailed form. The theory pays significant attention to differentiation and optimization of all the life cycle processes. The models proposed do not focus on a particular product or even a family of products - instead, they are oriented on the creation of a ubiquitous, broad and universal information infrastructure for design and life cycle engineering.

Concentrating on the theoretical aspects, this work contributed to the development of a general, interdisciplinary frame of design philosophy. To facilitate understanding of the main ideas, formal and computational details of the semiotic approach were not presented in the paper; the interested reader is however referred to [12] and [14]. Besides, some implementation issues as well as descriptions of case- and pilot- studies of the semiotic theory of evolutionary design can be found in [13] and [5].

In future work, the authors aim to build a complete classification of design and life cycle semiosis processes and investigate their properties. The further elaboration of the information infrastructure for the design semiosis services is also expected and should lead to the development of next generation information support tools and industrial technologies.

\section{ACKNOWLEDGEMENT}

This research has been supported in part by the Japan Society for the Promotion of Science (project No 96P00702). Dr. Goossenaerts would like to acknowledge the support of the ESPRIT IMS Working Group, EP 21995. We thank Prof. John J. Mills for valuable comments on the draft.

\section{REFERENCES}

[1] Aerts, A.T.M., Szirbick, N.B, Goossenaerts, J.B.M. (2000). Flexible Infrastructure for Virtual Enterprises, In: DIISM'2000 (this volume) 
[2] Arciszewski, T., Michalski, R.S., Wnek, J. (1995). Constructive induction: The key to design creativity, In: Preprints "Computational Models of Creative Design," Third International Round-Table Conference, pp. 397-426. Heron Island, Australia

[3] Collected papers of Charles Sanders Peirce, (in 6 volumes). (1960). C. Hartshorne and P. Weiss (eds.), The Belknap Press of Harvard University Press, Cambridge, MA

[4] Goguen, J.A. (1999). An introduction to algebraic semiotics, with applications to user interface design, In: C. Nehaniv (ed.), Computation for Metaphor, Analogy and Agents, Springer Lecture Notes in Artificial Intelligence, vol. 1562, 242-291

[5] Goossenaerts, J.B.M. (1999). A Systematisation Framework for Legal Drafting and Norm Implementation in the Information Society. Preprint. Eindhoven University of Technology, the Netherlands

[6] Goossenaerts, J.B.M. (2000). Industrial Semiosis: Founding the deployment of the Ubiquitous Information Infrastructure. Computers in Industry, vol. 43, 189-201

[7] Goossenaerts, J.B.M., Thoben, K.-D. (1999). The Artefactual Wheel-Work and the Information Infrastructure, In: H. Yoshikawa, R. Yamamoto, F. Kimura, T. Suga, and Y. Umeda (eds.), Proceedings of the First International Symposium on Environmentally Conscious Design and Inverse Manufacturing “EcoDesign'99," pp. 674-679, Tokyo

[8] Heylighen, F. (1990). Non-Rational Cognitive Processes as Changes of Distinctions, Communication \& Cognition, vol. 23, No.2-3, 165-181

[9] van den Hoed, R. (1997). An exploration of approaches towards Sustainable Innovation and their implications on the product development process, In: Proceedings of The Greening of Industry Conference "Developing Sustainability: New Dialogue, New Approaches." University of California, Santa Barbara, CA

[10] Hubka, V., Eder, W.E. (1996). Design Science: Introduction to the needs, scope and organization of Engineering Design knowledge, Springer-Verlag, London

[11] Klein, R. (1998). A Knowledge Level Theory of Design and Engineering, In: G. Jacucci, G.J. Olling, K. Preiss, M.J. Wozny (eds.), Globalization of Manufacturing in the Digital Communication Era of the 21st Century: Innovation, Agility, and the Virtual Enterprise, pp. 271-286, Kluwer Academic Publishers

[12] Kryssanov V.V., Tamaki, H., Kitamura, S. (1999). Understanding Design Fundamentals: How Synthesis and Analysis Drive Creativity, Resulting in Emergence, In: K. Ueda (ed.), Proceedings of the International Workshop on Emergent Synthesis, pp. 37-48, Kobe

[13] Kryssanov, V.V., Goossenaerts, J., Goncharenko, I., Tamaki, H. (2000). A Semiotic theory of evolutionary design: concepts and an illustration. In: Proceedings of the 7 th CIRP International Seminar on Life Cycle Engineering, pp. 68-75, Tokyo

[14] Kryssanov, V.V., Tamaki, H., Ueda, K. (1999). Agents for Assessing Requirements in Dynamic Environments to Support Evolutionary Design, In: Proceedings of the 6th CIRP International Seminar on Life Cycle Engineering, pp. 151-160, Kingston, Canada

[15] van Onck, A. (1994). Design. Il Senso Delle Forme Dei Prodotti. Lupetti Editori di Comunicazione, Milan (in Italian)

[16] Suh, N.P. (1990). The Principles of Design. Oxford University Press, NY

[17] Tomiyama, T. (1999). The Post Mass Production Paradigm, In: H. Yoshikawa, R. Yamamoto, F. Kimura, T. Suga, and Y. Umeda (eds.), Proceedings of the First International Symposium on Environmentally Conscious Design and Inverse Manufacturing “EcoDesign'99," pp. 162-167, Tokyo

[18] Wouters, M.J.F., Sharman G.J., Wortmann H.C. (1999). Reconstructing the Sales and Fulfillment Cycle to Create Supply Chain Differentiation, International Journal of Logistics Management, vol. 10(2), 83-97

[19] Yoshikawa, H. (1981). General Design Theory and a CAD System, In: T. Sata and E.A. Warman (eds.), Man-Machine Communication in CAD/CAM, pp. 35-58, North-Holland 